\author{
Marquette University \\ e-Publications@Marquette
}

School of Dentistry Faculty Research and

Publications

Dentistry, School of

2017

\title{
Expression of MMP-13 in Human Temporomandibular Joint Disc Derangement and Osteoarthritis
}

Juliano Henrique Perotto

Pontifícia Universidade Católica do Paraná

Flavio Camejo

Pontifícia Universidade Católica do Paraná

Andrea Doetzer

Pontifícia Universidade Católica do Paraná

Luis Eduardo Almeida

Marquette University, luis.almeida@marquette.edu

Marina Azevedo

Pontificia Universidade Catolica do Parana

See next page for additional authors

Follow this and additional works at: https://epublications.marquette.edu/dentistry_fac

Part of the Dentistry Commons

\section{Recommended Citation}

Perotto, Juliano Henrique; Camejo, Flavio; Doetzer, Andrea; Almeida, Luis Eduardo; Azevedo, Marina; and Olandoski, Marcia, "Expression of MMP-13 in Human Temporomandibular Joint Disc Derangement and Osteoarthritis" (2017). School of Dentistry Faculty Research and Publications. 257.

https://epublications.marquette.edu/dentistry_fac/257 


\section{Authors}

Juliano Henrique Perotto, Flavio Camejo, Andrea Doetzer, Luis Eduardo Almeida, Marina Azevedo, and Marcia Olandoski

This article is available at e-Publications@Marquette: https://epublications.marquette.edu/dentistry_fac/257 
Marquette University

e-Publications@Marquette

\section{Dentistry Faculty Research and Publications/School of Dentistry}

This paper is NOT THE PUBLISHED VERSION; but the author's final, peer-reviewed manuscript. The published version may be accessed by following the link in the citation below.

CRANIO ${ }^{\circledR}$ : The Journal of Craniomandibular \& Sleep Practice, (2017). DOI. This article is (C) Taylor \& Francis and permission has been granted for this version to appear in e-Publications@Marquette. Taylor \& Francis does not grant permission for this article to be further copied/distributed or hosted elsewhere without the express permission from Taylor \& Francis.

\section{Expression of MMP-13 in Human Temporomandibular Joint Disc Derangement and Osteoarthritis}

\section{Juliano Henrique Perotto}

School of Health and Biosciences, Pontíficia Universidade Católica do Paraná, Curitiba, Brazil Flavio de Alcantara Camejo

School of Health and Biosciences, Pontíficia Universidade Católica do Paraná, Curitiba, Brazil Andrea Duarte Doetzer

School of Health and Biosciences, Pontíficia Universidade Católica do Paraná, Curitiba, Brazil Luis Eduardo Almeida

School of Dentistry, Marquette University, Milwaukee, WI

Marina Azevedo

School of Health and Biosciences, Pontíficia Universidade Católica do Paraná, Curitiba, Brazil Márcia Olandoski

School of Health and Biosciences, Pontíficia Universidade Católica do Paraná, Curitiba, Brazil Lucia Noronha

School of Health and Biosciences, Pontíficia Universidade Católica do Paraná, Curitiba, Brazil 


\section{Paula Cristina Trevilatto}

School of Health and Biosciences, Pontíficia Universidade Católica do Paraná, Curitiba, Brazil

\section{Abstract}

Objective: MMP-13 performs digestion of collagen, which is a primary component of the temporomandibular joint (TMJ) articular disc. This study evaluated the expression of MMP-13 in patients with anterior disc displacement with (ADDwR) and without reduction (ADDwoR), and in the presence of TMJ osteoarthrosis.

Methods: Thirty-nine human temporomandibular joint disc samples were collected and divided in two ways: ADDwR (21 samples), ADDwoR (10 samples), and a control group (8 samples); and with osteoarthrosis (10 samples) and without osteoarthrosis (29 samples). Immunostaining of the TMJ discs was statistically compared between the groups.

Results: There was no statistically significant difference for the area of MMP-13 immunostaining between the control group, ADDwR, and ADDwoR, nor between groups with and without osteoarthrosis.

Conclusion: This study suggests MMP-13 is not significantly involved in collagen degradation in human TMJ disc displacement or osteoarthrosis.

Keywords: Temporomandibular disorder, disc derangement, osteoarthrosis

\section{Introduction}

Temporomandibular joint derangement (TMD) is a common disease that affects many people around the world, with a major prevalence in females ${ }^{[1-4]}$. The most commonly found disorder in the temporomandibular joint (TMJ) is the abnormal positioning of the articular disc ${ }^{[1,5-8]}$. The two most frequent types of disc displacement are anterior disc displacement with (ADDwR) and without reduction (ADDwoR). In ADDwR, the disc returns to its normal position during mouth opening, and it goes back to its displaced position when the mouth closes completely, which is clinically represented by an articular click. Hence, in ADDwoR, the disc is unable to return to its normal position, which leads to a decrease in mouth opening and is usually painful [9,10].

The disc displacement may contribute to the progression of TMJ derangement and could lead to osteoarthrosis of the TMJ, which is characterized by the abnormal remodeling of osseous structures of the joint, frequently accompanied by pain, limiting functional capability [11-14].

Previous studies have shown that alteration of the disc structure may impact the expression of some mediators related to apoptosis and inflammatory process, and they could be associated with TMD progression ${ }^{[15-26]}$. The articular disc of the TMJ is formed basically by proteoglycan aggregates and collagen fibers that are composed mainly of type 1 and 2 collagen; its physiologic maintenance is through a balance between degradation of collagen fibers performed by matrix metalloproteinase (MMP) and their inhibitors, tissue inhibitor of matrix metalloproteinase (TIMP) [26-28]. 
In this study, the hypothesis was that MMP-13 might be involved in the progression of TMD. Therefore, the present investigation was designed to evaluate, through immunohistochemistry, the expression of MMP-13 in TMJ articular discs of ADDwR and ADDwoR patients. Moreover, the presence of TMJ osteoarthrosis was also investigated to better understand the relationship between TMD advanced stages and MMP-13 expression.

\section{Materials and methods}

Sample selection

A sample of 39 temporomandibular joint discs was collected from 27 patients, with a mean age of 33.59 years old (17 to 57 years), for study at the Evangelico School Hospital, Curitiba, in the Southern region of Brazil (Table 1). This study was approved by the Ethical Committee on Research at Pontifical Catholic University of Paraná, according to Resolution 196/96 of the National Health Council and approved under registration number 104. Subjects were not included in the study if presenting: use of orthodontic appliances, chronic usage of antiinflammatory drugs, history of diabetes, hepatitis, HIV infection, immunosuppressive chemotherapy, history of any disease known to severely compromise immune function, current pregnancy or lactation, dentofacial deformity, major jaw trauma, previous TMJ surgery, or previous steroid injection in the TMJ. 


\begin{tabular}{|c|c|c|c|c|c|c|}
\hline \multirow[b]{2}{*}{ Patient } & \multirow[b]{2}{*}{ Gender } & \multirow[b]{2}{*}{ Age (yrs) } & \multirow[b]{2}{*}{ Diagnosis } & \multicolumn{2}{|c|}{ Affected side } & \multirow[b]{2}{*}{ Wilkes Stage } \\
\hline & & & & Right & Left & \\
\hline 1 & $\mathrm{~F}$ & 33 & ADDwR & $x$ & & III \\
\hline 2 & M & 27 & CFx & & $\mathrm{x}$ & \\
\hline 3 & M & 33 & CFx & $\mathrm{x}$ & & \\
\hline 4 & $\mathrm{~F}$ & 26 & ADDwR & $x$ & & III \\
\hline 4 & $\mathrm{~F}$ & 26 & ADDwrR & & $x$ & III \\
\hline 5 & $\mathrm{~F}$ & 43 & $\mathrm{CH}$ & & $x$ & \\
\hline 6 & $\mathrm{~F}$ & 17 & $\mathrm{CH}$ & & $x$ & \\
\hline 7 & $\mathrm{~F}$ & 30 & ADDwr & & $x$ & III \\
\hline 8 & $\mathrm{~F}$ & 25 & ADDwR & & $x$ & III \\
\hline 8 & $\mathrm{~F}$ & 25 & ADDwR & $\mathrm{x}$ & & III \\
\hline 9 & $\mathrm{~F}$ & 37 & ADDwR & $x$ & & III \\
\hline 9 & $F$ & 37 & ADDwR & & $x$ & III \\
\hline 10 & M & 42 & CFx & $x$ & & \\
\hline 11 & $\mathrm{~F}$ & 20 & ADDwr & & $x$ & III \\
\hline 12 & $\mathrm{~F}$ & 23 & ADDwor R & & $x$ & v \\
\hline 12 & $\mathrm{~F}$ & 23 & ADDwor & $x$ & & v \\
\hline 13 & $\mathrm{~F}$ & 36 & ADDwR & $\mathrm{x}$ & & III \\
\hline 13 & $\mathrm{~F}$ & 36 & ADDwR & & $x$ & III \\
\hline 14 & $\mathrm{~F}$ & 38 & ADDwR & $\mathrm{x}$ & & III \\
\hline 14 & $\mathrm{~F}$ & 38 & ADDwR & & $x$ & III \\
\hline 15 & $\mathrm{~F}$ & 22 & ADDwR & $x$ & & III \\
\hline 15 & $\mathrm{~F}$ & 22 & ADDwR & & $x$ & III \\
\hline 16 & $\mathrm{~F}$ & 26 & ADDwor & $x$ & . & IV \\
\hline 16 & $\mathrm{~F}$ & 26 & ADDwoR & & $\mathrm{x}$ & IV \\
\hline 17 & $\mathrm{~F}$ & 32 & ADDwor & & $x$ & IV \\
\hline 17 & $\mathrm{~F}$ & 32 & ADDwor & $x$ & & $\mathrm{v}$ \\
\hline 18 & $\mathrm{~F}$ & 45 & ADDwor R & $x$ & & v \\
\hline 19 & $\mathrm{~F}$ & 35 & ADDwor & & $x$ & IV \\
\hline 19 & $\mathrm{~F}$ & 35 & ADDwoR & $x$ & 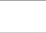 & IV \\
\hline 20 & $\mathrm{~F}$ & 24 & ADDwor & & $x$ & $\mathrm{v}$ \\
\hline 21 & $\mathrm{~F}$ & 34 & ADDwR & & $x$ & III \\
\hline 22 & $\mathrm{~F}$ & 57 & ADDwR & & $x$ & III \\
\hline 23 & $\mathrm{~F}$ & 18 & |CFx & & $x$ & \\
\hline 23 & $\mathrm{~F}$ & 18 & CFx & $x$ & & \\
\hline 24 & M & 46 & ADDwR & & $x$ & III \\
\hline 24 & M & 46 & ADDwR & $x$ & & III \\
\hline 25 & $\mathrm{~F}$ & 40 & $\mathrm{CH}$ & & $x$ & \\
\hline 26 & $\mathrm{~F}$ & 56 & ADDwR & $x$ & & III \\
\hline 27 & $\mathrm{~F}$ & 42 & ADDwR & $\mathrm{x}$ & & III \\
\hline
\end{tabular}

Table 1. Baseline clinical characteristics of the control and study groups with and without TMJ dysfunction, according to Wilkes stage.

Subjects completed personal medical history questionnaires and, within a protocol approved by an Institutional Review Board, signed a consent form after being advised of the nature of the study. All patients were asked to complete a pain questionnaire, and a clinical examination was performed according to Clinical Practice Guidelines for TMJ surgery of the American Association of Oral Maxillofacial Surgeons. The patients were considered to be affected and were treated surgically when presenting painful clinical signs of disc displacement after unsuccessful nonsurgical treatment for at least six months. 
Out of the eight control joints, five were condyle fractures (CFX), confirmed by radiographs and CT scan; the patients had to undergo surgery for the fracture reduction. Three joints displayed active condyle hyperplasia $(\mathrm{CH})$, diagnosed by radiographs. All patients had a panorex; the patients with condyle fracture had a CT scan, and most of the patients in the study group had an MRI.

The sample was divided as follows:

(1) Joints without any signs of disc displacement (control group; $n=8$ disc specimens);

(2) Joints presenting anterior disc displacement with reduction (ADDwR; $n=21$ disc specimens);

(3) Joints presenting anterior disc displacement without reduction (ADDwoR; $n=10$ disc specimens).

Joints were clinically categorized according to the presence or absence of disc displacement and, secondarily, according to the presence or absence of osteoarthrosis (according to Wilkes classification) [20].

Joints' selection for osteoarthrosis analysis was based on the primary diagnosis of severe TMJ internal derangement (ID). The stages of TMJ ID were classified into mild, intermediate, and severe, according to Wilkes classification, based on clinical, surgical, and pathological stages [29]. Mild ID (Wilkes stage III) is characterized by simple disc displacement without any morphological alteration of the disc and with or without osseous shift. The intermediate stage (Wilkes stage IV) is characterized by disc displacement and morphological deformity and/or osseous remodeling changes. Severe derangement (Wilkes stage $V$ ) is characterized by perforations of the disc attachments and osseous shift and/or osteoarthrosis changes (sclerosis, osteophyte formation, articular surface flattening, depression and/or cystic alterations) [29]. Joints from the control group and those classified as Wilkes III were considered not presenting osteoarthrosis, and joints classified as Wilkes IV or $V$ were included in the osteoarthrosis group, as follows:

(1) Joints without osteoarthrosis (control group + Wilkes stage III; $n=29$ disc specimens);

(2) Joints with osteoarthrosis (Wilkes stage IV and V; $n=10$ disc specimens).

Table 1 shows the baseline characteristics of the sample.

Surgical technique

TMJ surgery was performed by the same surgeon, according to the technique described by Mehra and Wolford [30].

First, the displaced disc was freed by the surgeon entering the upper and lower joint spaces and lysing adhesions. At this point, a small hole was placed through the lateral-posterior pole of the condyle from a posterior to anterior direction. The Mitek bone-cleat introducer was 
inserted and pushed into the bone, where two small coils unlocked and attached the cleat to the inner surface of the condyle cortical bone. A non-resorbable 2-0 or 3-0 suture was placed through the hole and through the disc at the junction of the posterior and intermediate bands, and the disc was tied down to the condylar neck. The deformity of the disc precluded repositioning it into the ideal position, and re-contouring the thickened disc with a scalpel was necessary (this scalpelled material constitutes the specimen).

This procedure was conducted for all patients with disc displacement and the control group. In the CFx patients, the disc displaced by fracture was repositioned, and in the $\mathrm{CH}$ patients, the disc was sutured to prevent disc displacement caused by the gap that was created after the high condylectomy. Postsurgical physical therapy was indicated at the discretion of the surgeon.

Histological sections obtained by scalpel of disc excess were prepared for observation of the in situ expression of MMP-13 by immunohistochemistry.

\section{Immunohistochemistry}

The TMJ disc sections were deparaffinized with xylol $(2 \times 10 \mathrm{~min})$ and rehydrated with absolute ethylic alcohol $(3 \times 1 \mathrm{~min})$ and $80 \%$ ethylic alcohol $(1 \times 1 \mathrm{~min})$. Endogenous peroxidase activity was quenched by treatment with $\mathrm{H}_{2} \mathrm{O}_{2}$ (5\% in methanol) for 10 min. Target Retrieval Solution ${ }^{\mathrm{TM}}$ (Dako, DK-2600 Glostrup, Denmark) was used prior to slide staining for heat-inducing epitope retrieval (for formalin-fixed, paraffin-embedded material), according to the manufacturer's instructions. The sections were incubated with monoclonal MMP-13 antibody (Abcam PLC, Cambridge, UK), diluted 1:50 in phosphate-buffered saline (PBS), 0.1\% bovine serum albumin (BSA). For negative controls, the primary antibody was not added. PBS was used instead. The secondary antibody, AdvanceTM (Dako, DK-2600 Glostrup, Denmark), was applied for 30 min, according to the manufacturer's instruction.

The immunoreactions were visualized by incubating the sections using 3,3' diaminobenzidine (DAB) chromogen (OriGene, Rockville, MD, USA), (1 drop in $1 \mathrm{~mL}$ distilled water). The sections were lightly counterstained with Harris haematoxylin for $5 \mathrm{~min}$, and finally mounted. Immunostaining was considered to be specific to MMP-13 because immunoreactivity was not observed in the negative controls.

The color morphometry method was used to analyze the anti-MMP-13 immunostained area in the TMJ disc tissue. For this purpose, images of consecutive fields were captured by a 20x objective lens coupled with the BX50 Olympus microscope with a Sony camera, Model DXC107A, and image analysis was performed with specific software called Image Pro Plus software (Media Cybernetics Inc., Rockville, MD, USA). This software allows an observer to select and paint the positive areas to obtain an image model and make the mask for the other stained slides, the area of the positive reaction being automatically calculated. This procedure was performed by a single examiner in a blind manner. The data were entered into a spreadsheet, and Microsoft Excel (Redmond, WA, USA) was used to obtain the statistical analysis. The variable area was measured in square micrometers $\left(\mu \mathrm{m}^{2}\right)$ and was obtained with the mean of all positive areas. 


\section{Statistical analysis}

To compare the groups (control, ADDwR and ADDwoR) regarding area, the non-parametric Kruskal-Wallis analysis was considered. To compare groups with and without osteoarthrosis, the non-parametric Mann-Whitney test was employed. To meet the symmetric condition of the variable, data of area were previously submitted to a logarithmic transformation. The $p$ value $<0.05$ was considered statistically significant. Data were analyzed with IBM SPSS Statistics v.20.0 software (IBM Corporation, Armonk, NY, USA).

\section{Results}

Expression of MMP-13 was observed at cytoplasm level.

\section{Expression of MMP-13 in TMJ sample ADDwR, ADDwoR and control}

Significant differences were not found in the expression of MMP-13 in TMJ discs between the three groups for the variable area $(p=0.288)$ (Table 2).

\begin{tabular}{|c|c|c|c|c|c|c|c|c|}
\hline Variable & Group & $n$ & Mean & Median & Minimum & Maximum & Standard deviation & $p$-value* \\
\hline Area & Control & 8 & 17428 & 7292 & 1432 & 52714 & 19534 & \\
\hline & With reduction & $\mid 21$ & 11155 & 5947 & 108 & 54225 & 13058 & \\
\hline & Without reduction & 10 & 6364 & 2466 & 418 & 19862 & 6951 & 0.288 \\
\hline
\end{tabular}

Table 2. MMP-13 area of immunostaining ( $\mu \mathrm{m} 2)$ in the discs of the control and study groups with and without TMJ dysfunction.

Expression of MMP-13 in TMJ discs of patients with and without osteoarthrosis

All joints with ADDwoR presented osteoarthrosis. On the other hand, almost all joints without osteoarthrosis presented ADDwR.

Statistically significant differences were not found in the expression of MMP-13 in TMJ discs between the groups with and without osteoarthrosis for the variable area $(p=0.185)$ (Table 3). 


\begin{tabular}{|c||l||c||c||c||c||c||c||c||c|}
\hline \multicolumn{1}{|c|}{ Variable } & \multicolumn{1}{|c|}{ Group } & $\boldsymbol{n}$ & Mean & Median & Minimum & Maximum & \multicolumn{1}{|c|}{ Standard deviation } \\
\hline \hline Area & With osteoarthrosis & 10 & 6364 & 2466 & 418 & 19862 \\
\hline \hline & Without osteoarthrosis & 29 & 12885 & 6933 & 108 & 5951 \\
\hline
\end{tabular}

Table 3. Differences between groups with and without osteoarthrosis, with respect to area of in situ expression $(\mu \mathrm{m} 2)$ of MMP-13 cytokine.

\section{Discussion}

In the last few years, the expression of matrix metalloproteases and some others markers, such as Fas Ligand (FasL) and ADAM metallopeptidase domain 17 (ADAM-17), were shown in the progression of TMJ ID using TMJ discs and synovial fluid [15-26].

The articular disc of the TMJ is formed by proteoglycan aggregates and collagen types 1 and 2. But the TMJ's disc is a fibrocartilage, different from other joints in the body (hyaline cartilage), and this disc is composed mostly of type 1 collagen rather than type 2 , whereas hyaline cartilage has no type 1 collagen $[5,10,13$. A balance between degradation of collagen fibers performed by matrix metalloproteinase (MMP) and their tissue inhibitor of metalloproteinase (TIMP), are responsible for functional remodeling of the TMJ [21,26-28].

MMPs have been reported to be involved in the cleavage of cell surface receptors and the release of apoptotic ligands (such as the Fas ligand). They play an important role in tissue remodeling associated with various physiological or pathological processes such as morphogenesis, angiogenesis, tissue repair, cirrhosis, arthritis, and metastasis [21,27,28].

Loreto et al. [21] showed that MMP-7 and MMP-9 are expressed in arthritic joints and provided evidence of a role for those MMPs in TMJ ID disc damage, with higher expression being detected in the posterior rather than in the anterior and intermediate bands of ADDwR and ADDwoR discs.

Almeida et al. [24], in a study using MMP-2 and MMP-9, did not find a statistically significant difference between the variable area of MMP-9 expression in the disc with or without disc displacement, as determined by immunohistochemical analysis. However, an elevation of MMP-2 expression in the discs of patients with ADDwoR (more severe alteration) was identified.

One of the most important metalloproteinases involved in degradation of collagen within the cartilage is MMP-13 (collagenase-3), a metalloproteinase of the collagenase group, which performs preferential digestion of type 2 collagen over type 1 and 3 collagens [26-28]. Leonardi et al. [26] found correlation of an increased MMP-13 immunoreactivity in TMJ diseased disc tissue with the increased severity of the histopathological changes; however that study was performed with cadavers' discs.

In this study, the hypothesis is that MMP-13 may be involved in the progression of TMD, since other studies have already demonstrated the correlation of MMPs with this progression. 
Significant differences were not observed between the groups control and with or without anterior disc reduction of the articular disc, with respect to the variable area for the expression of MMP-13 measured by immunohistochemical examination. This lack of expression could be due to the composition of the TMJ disc, which is mainly collagen type 1 , and MMP-13 is less effective at breaking down type 1 collagen than type 2 collagen.

Osteoarthrosis is characterized by deterioration and abrasion of articular cartilage. Some papers have demonstrated the association of MMPs (MMP-7, MMP-9, MMP-13) with osteoarthrosis, an inflammatory disorder of movable joints, in the degenerative process of joints included in the TMJ [21,27,28].

Schlopov et al. [31] suggested a key role of MMP-13 and MMP-8, as well as MMP-1 in osteoarthrosis.

This study has some limitations, such as the restrictive sample size; however this still seems to be the biggest sample size so far in TMJ studies, to the authors' knowledge. This reduced number refers to the fact that only a low number of patients must be submitted to surgical treatment.

In this study, significant differences between the groups with and without osteoarthrosis with respect to the variable area for the expression of MMP-13 measured by immunohistochemical examination were not observed.

\section{Conclusion}

In conclusion, the expression of MMP-13 was not associated with TMD and osteoarthrosis in the study population.

\section{Disclosure statement}

No potential conflict of interest was reported by the authors.

\section{Acknowledgments}

Flavio de Alcantara Camejo would like to thank Pontifical Catholic University of Paraná (PUCPR) for the PhD scholarship provided. Andrea Duarte Doetzer was supported by CAPES/PROSUP and DAAD/CNPq scholarships.

\section{References}

1 Murphy MK, MacBarb RF, Wong ME, et al. Temporomandibular disorders: a review of etiology, clinical management, and tissue engineering strategies. Int J Oral Maxillofac Implants. 2013 Nov-Dec;28(6):e393-e414.10.11607/jomi.te20

2 Martins-junior RL, Palma AJ, Marquardt EJ, et al. Temporomandibular disorders: a report of 124 patients. J Contemp Dent Pract. 2010;11:071-78. 
3 Solberg WK, Woo MW, Houston JB. Prevalence of mandibular dysfunction in young adults. J Am Dent Assoc. 1979;98:25-34.10.14219/jada.archive.1979.0008

4 Warren MP, Fried JL. Temporomandibular disorders and hormones in women. Cells Tissues Organs. 2001;169:187-192.10.1159/000047881

5 Westesson PI, Larheim TA, Tanaka H. Posterior disc displacement in the temporomandibular joint. J Oral Maxillofac Surg. 1998;56:1266-1273.10.1016/S02782391(98)90606-1

6 Blankestijn J, Boering G. Posterior dislocation of the temporomandibular disc. Int J Oral Surg. 1985;14:437-443.10.1016/S0300-9785(85)80076-4

7 Huddleston Slater JJ, Lobbezoo F, Hofman N, et al. Case report of a posterior disc displacement without and with reduction. J Orofac Pain. 2005;19:337-342.

8 Chiba M, Watanabe N, Echigo S. Longitudinal MRI follow-up of non-reducible posterior disc displacement accompanied by bone marrow oedema in the mandibular condyle. Dentomaxillofac Radiol. 2007;36:304-307.10.1259/dmfr/64232609

9 Pérez del Palomar DP, Doblaré M. An accurate simulation model of anteriorly displaced TMJ discs with and without reduction. Med Eng Phys. 2007;29:216226.10.1016/j.medengphy.2006.02.009

10 Okeson JP. Management of temporomandibular disorders and occlusion. Vol. 294. St Louis: Mosby-Year book, Inc; 1993. p. 409-477.

11 Wang XD, Zhang JN, Gan YH, et al. Current understanding of pathogenesis and treatment of TMJ osteoarthritis. J Dent Res. 2015 Mar 5. pii: 0022034515574770. [Epub ahead of print] Review.

12 Henderson SE, Tudares MA, Tashman S, et al. Decreased temporomandibular joint range of motion in a model of early osteoarthritis in the rabbit. J Oral Maxillofac Surg. 2015 Mar 25. pii: S0278-2391(15)00334-1. doi: 10.1016/j.joms.2015.03.042 [Epub ahead of print].

13 de Bont LGM, Stegenga B. Pathology of temporomandibular joint internal derangement and osteoarthrosis. Int J Oral Maxillofac Surg. 1993;22:71-74.10.1016/S09015027(05)80805-7

14 Segù M, Politi L, Galioto S, et al. Histological and functional changes in retrodiscal tissue following anterior articular disc displacement in the rabbit: review of the literature. Minerva Stomatol. 2011 Jul-Aug;60(7-8):349-358.

15 Leonardi R, Almeida LE, Rusu M, et al. Tumor necrosis factor-related apoptosis-inducing ligand expression correlates to temporomandibular joint disc degeneration. J Craniofac Surg. 2011;22:504-508.10.1097/SCS.0b013e3182087394

16 Leonardi R, Migliore MR, Almeida LE, et al. Limited fatty infiltration due to apoptosis in human degenerated temporomandibular joint discs: an immunohistochemical study. J Craniofac Surg. 2010;21:1508-1511.10.1097/SCS.0b013e3181ef2dc9

17 Leonardi R, Almeida LE, Trevilatto P, et al. Occurrence and regional distribution of TRAIL and DR5 on temporomandibular joint discs: comparison of disc derangement with and without reduction. Oral Surg Oral Med Oral Pathol Oral Radiol Endod. 2010;109:244251.10.1016/j.tripleo.2009.09.028

18 Loreto C, Almeida LE, Trevilatto P, et al. Apoptosis in displaced temporomandibular joint disc with and without reduction: an immunohistochemical study. J Oral Pathol Med. 2011;40:103-110.10.1111/jop.2010.40.issue-1 
19 Loreto C, Almeida LE, Migliore MR, et al. TRAIL, DR5 and caspase 3-dependent apoptosis in vessels of diseased human temporomandibular joint disc. An immunohistochemical study. Eur J Histochem. 2010;54:e40.10.4081/ejh.2010.e40

20 Loreto C, Musumeci G, Leonardi R. Chondrocyte-like apoptosis in temporomandibular joint disc internal derangement as a repair-limiting mechanism. An in vivo study. Histol Histopathol. 2009;24:293-298.

21 Loreto C, Leonardi R, Musumeci G, et al. An ex vivo study on immunohistochemical localization of MMP-7 and MMP-9 in temporomandibular joint discs with internal derangement. Eur J Histochem. 2013 Apr 15;57(2):e12.10.4081/ejh.2013.e12

22 Imirzalioğlu P, Uçkan S, Güler N, et al. Synovial apoptosis in temporomandibular joint disc displacement without reduction. Oral Surg Oral Med Oral Pathol Oral Radiol Endod. 2009;108:693-698.10.1016/j.tripleo.2009.05.030

23 De Alcântara Camejo F, Almeida LE, Doetzer AD, et al. FasL expression in articular discs of human temporomandibular joint and association with osteoarthrosis. J Oral Pathol Med. 2014;43(1):69-75.10.1111/jop.2013.43.issue-1

24 Almeida LE, Caporal K, Ambros V, et al. Immunohistochemical expression of matrix metalloprotease-2 and matrix metalloprotease- 9 in the discs of patients with temporomandibular joint dysfunction. J Oral Pathol Med. 2015 Jan;44(1):75-79. Epub 2014 Jul 28.10.1111/jop.2014.44.issue-1

25 Leonardi R, Crimi S, Almeida LE, et al. ADAMTS-4 and ADAMTS-5 expression in human temporomandibular joint discs with internal derangement, correlates with degeneration. J Oral Pathol Med. 2014 Dec 5. doi: 10.1111/jop.12295 [Epub ahead of print].

26 Leonardi R, Loreto C, Barbato E, et al. MMP-13 (collagenase 3) localization in human temporomandibular joint discs with internal derangement. Acta Histochem. 2008;110(4):314-318. Epub 2008 Feb 8.10.1016/j.acthis.2007.11.010

27 Wang YL, Li XJ, Qin RF, et al. Matrix metalloproteinase and its inhibitor in temporomandibular joint osteoarthrosis after indirect trauma in young goats. $\mathrm{Br} \mathrm{J}$ Oral Maxillofac Surg. 2008 Apr;46(3):192-197. Epub 2007 Dec 31.10.1016/j.bjoms.2007.10.007

28 Ferreira LM, Moura AF, Barbosa GA, et al. Do matrix metalloproteinases play a role in degenerative disease of temporomandibular joint? A systematic review. CRANIO ${ }^{\text {. }}$. 2015 Feb 3:2151090314Y0000000034. [Epub ahead of print].

29 Wilkes C. Arthrography of the temporomandibular joint inpatients with the TMJ paindysfunction syndrome. Minn Med. 1978;61:645-652.

30 Mehra P, Wolford LM. Use of the Mitek anchor in temporomandibular joint discrepositioning surgery. Proc (Bayl Univ Med Cent). 2001;14:22-26.

31 Shlopov BV, Gumanovskaya ML, Hasty KA. Autocrine regulation of collagenase 3 (matrix metalloproteinase 13) during osteoarthritis. Arthritis Rheum. 2000 Jan;43(1):195205.10.1002/(ISSN)1529-0131 